\title{
Mild traumatic brain injury and posttraumatic stress disorder: Investigation of visual attention in Operation Iraqi Freedom/Operation Enduring Freedom veterans
}

\author{
Kristen Barlow-Ogden, MA; ${ }^{*}$ William Poynter, PhD $^{\mathbf{2}}$ \\ ${ }^{1}$ Surgical Service, Charles George Department of Veterans Affairs Medical Center, Asheville, NC; ${ }^{2}$ Department of \\ Psychology, Western Carolina University, Cullowhee, NC
}

\begin{abstract}
Mild traumatic brain injury (mTBI) and posttraumatic stress disorder (PTSD) are prevalent dual impairments in veterans returning from the wars in Iraq and Afghanistan. Attention problems are a common self-reported complaint of veterans with $\mathrm{mTBI}$, but relatively few studies have investigated the types and levels of behavioral attentional deficits present in veterans with mTBI and PTSD. The purpose of this study was to compare visual attentional performance between samples of veterans with both mTBI and PTSD (mTBI+PTSD), PTSD only, and a control group. Overall, the attentional responses of the mTBI+PTSD group were slower than those of the PTSD and control groups. The response times were also more variable, suggesting difficulty with attentional vigilance. Additionally, we found evidence of hemispheric asymmetries in attentional performance. Participants with mTBI+PTSD were less efficient in orienting visual attention to stimuli flashed to the left visual field (LVF), suggesting a right hemisphere deficit. Overall, we found that veterans who had sustained an mTBI and had a coexisting PTSD diagnosis displayed longer response times and were less accurate than the PTSD and control groups, especially when cues were presented to the LVF.
\end{abstract}

Key words: attention, blast event, brain concussion, hemispheric asymmetries, lateralized attention network task, mild traumatic brain injury, OIF/OEF veterans, polytrauma, posttraumatic stress disorder, vision.

\section{INTRODUCTION}

Mild traumatic brain injury (mTBI) and posttraumatic stress disorder (PTSD) have been named the "invisible wounds" of combat-related injury [1]. In addition, mTBI has been called the "signature injury" of the Iraq war [2]. "We are going to have a large population of individuals with significant brain impairment who are going to have difficulty navigating through every day life," said William Perry, president of the National Academy of

Abbreviations: $\mathrm{ADHD}=$ attention deficit/hyperactivity disorder, ANOVA = analysis of variance, ANT = attention network task, CPRS = Computerized Patient Record System, DVBIC = Defense and Veterans Brain Injury Center, IRB = institutional review board, LANT = lateralized attention network task, LH = left hemisphere, $\mathrm{LVF}=$ left visual field, $\mathrm{mTBI}=$ mild traumatic brain injury, OEF = Operation Enduring Freedom, OIF = Operation Iraqi Freedom, PHQ-2 = Patient Health Questionnaire-2, PTSD = posttraumatic stress disorder, $\mathrm{RH}=$ right hemisphere, $\mathrm{RT}=$ response time, $\mathrm{RVF}=$ right visual field, $\mathrm{SD}=$ standard deviation, TBI $=$ traumatic brain injury, $\mathrm{VA}=$ Department of Veterans Affairs, VAMC $=$ Department of Veterans Affairs medical center, $\mathrm{VF}=$ visual field.

*Address all correspondence to Kristen Barlow-Ogden, MA; Charles George VA Medical Center, Eye Clinic, 1100 Tunnel Rd, Asheville, NC 28801; 828-298-7911, ext 15620; fax: 828-299-5872. Email: kristen.barlow@va.gov http://dx.doi.org/10.1682/JRRD.2010.09.0188 
Neuropsychology [2]. The exact proportion of troops who have mTBI is not known, although it has been reported to be as high as 18 percent [3]. Many overlapping symptoms are common to traumatic brain injury (TBI) and PTSD diagnoses, which causes problems in the management of clinical care for veterans returning from the wars in Iraq and Afghanistan. Many providers are faced with the challenges of treating these veterans who have reported dual impairments when they return to civilian life. Recent reports suggest that the rate of PTSD in returning Operation Iraqi Freedom (OIF)/Operation Enduring Freedom (OEF) veterans is similar to the rate of TBI and that 37 to 44 percent of those with a possible TBI may also have PTSD [4]. TBI and PTSD share a number of nonspecific symptoms, such as concentration difficulties, irritability, impaired decision-making abilities, and memory problems [1]. Attention problems are also common complaints of TBI and PTSD veterans. In a study of 66 TBI patients who had completed tours of military duty in Iraq or Afghanistan, more than 50 percent reported problems with attention [5]. However, relatively few studies of returning veterans with TBI and/or PTSD have investigated whether these veterans actually manifest attentional deficits on behavioral measures of attention. Therefore, the goal of the present study was to use an objective behavioral measure of attention to investigate attentional performance in veterans with mTBI and PTSD. Many studies have investigated attentional performance deficits in patients with TBI [6-11]. But arriving at a general conclusion regarding the effects of TBI on attentional function is difficult because these studies have used patients with different degrees of TBI (mild to severe) and injuries of different recency (days to years old). Additionally, a wide range of behavioral attention tasks has been used to measure a variety of attentional functions, including orienting of attention (spatial cueing paradigms), maintaining alertness of attention (vigilance tasks), and executive mechanisms of attention (selective attention tasks). These different types of attentional functioning are considered to involve three networks of attention, labeled by Fan and colleagues [12] as "alerting," "orienting," and "executive.” TBI-associated attentional deficits that involve sustained attention, or attentional vigilance, represent deficits in the alerting network. Problems shifting spatial attention (e.g., failure to benefit from spatial cueing in the classic Posner paradigm) represent deficits in the orienting network. Problems dividing attention in dual-task paradigms and problems selectively focusing attention on one task or stimulus and ignoring distracting stimuli represent deficits in the executive network. Van Donkelaar et al. used the attention network task (ANT) to measure alerting, orienting, and executive attentional functions in subjects who had sustained mTBI (concussion) from intramural sports or recreational activities [10]. They tested the participants, who were referred within $2 \mathrm{~d}$ of the injury, and found that participants with mTBI were slower overall than controls in reacting to target appearance, but also less efficient in orienting attention to the target and resolving visual conflict between the target and distracting flanker stimuli (executive function). Van Donkelaar et al.'s results indicate that the orienting and executive components of attention were negatively affected by concussion; however, the alerting component was unaffected [10]. Halterman et al. studied the time course of recovery from attentional deficits following mTBI in very recent TBI injuries (less than $2 \mathrm{~d}$ ) and found that mTBI patients performed significantly worse than control subjects on measures of the orienting and executive functions of attention on the first day of testing, but the orienting function returned to control levels within the $30 \mathrm{~d}$ test period, whereas the executive function did not [13]. No effect was observed for the alerting function across the study period. We are aware of only one study that has examined hemispheric asymmetries in attentional performance among TBI patients, and this study used patients with severe TBI [9]. Using a Posner spatial-cueing paradigm in which spatial cues were presented before visual target presentation, the investigators found that TBI patients were significantly slower to shift (orient) attention to the left visual field (LVF), therefore indicating a right hemisphere $(\mathrm{RH})$ deficit.

This RH orienting deficit has also been found in studies of subjects with attention deficit disorder and also nondisabled subjects who self-report high levels of attention problems in everyday activities [14]. Several studies have provided evidence that patients with attention deficit/ hyperactivity disorder (ADHD) exhibit worse attentional deficits for visual information presented in the LVF versus right visual field (RVF), indicating a RH deficit [1517]. Furthermore, a recent study found that non-ADHD individuals who self-report relatively high levels of attentional problems in everyday activities also exhibit an $\mathrm{RH}$ deficit in attentional tasks [14]. So, we were interested in comparing the performance of mTBI subjects in our sample who complained of attention problems with the performance of those that did not. We found only a few 
studies of attentional deficits in mTBI patients whose injuries were relatively old ( 1 to $5 \mathrm{yr}$ ). One such study, Cremona-Meteyard et al. [7], found persistent deficits in orienting visual attention in TBI patients $1-5$ yr post injury.

In general, studies of the effects of TBI on attentional function do not present an entirely consistent pattern of attentional deficits, but more often than not, TBI patients perform slower on behavioral tasks [6,10-11]. Additionally but less uniformly, TBI patients sometimes show deficits in selective/focused attention (executive function) depending on the specific task used [10,18-19], attentional vigilance (alerting function) with tasks that require sustained attention [8,11], and shifting spatial attention (orienting function) [7,9-10,13].

Attentional problems are also a common complaint of patients with a PTSD diagnosis. Leskin and White recently reported on three aspects of attentional network efficiency in PTSD [20]. Using the ANT initially developed by Fan et al. [12], they found that PTSD participants were slower in responding to incongruent flankers but not congruent flankers than were subjects without a PTSD diagnosis. These results suggest that PTSD subjects are specifically impaired in inhibiting irrelevant information, a function of the executive attentional network. Vasterling et al. studied attention, learning, and memory performances of Vietnam veterans with PTSD and measured four components of attention: (1) focusexecutive, (2) sustain, (3) shift, and (4) encode [21]. Vasterling et al.'s results suggest that veterans with PTSD differed significantly from those without PTSD on tasks measuring focused and sustained attention [21]. PTSD veterans responded to fewer correct cognitive performance test stimuli and performed less efficiently on the digit span subtest.

As mentioned previously, a high percentage of OIF/ OEF troops are diagnosed with PTSD and mTBI. Although the exact percentage of comorbidity in veterans remains unclear, cognitive, emotional, and behavioral functions significantly overlap between the diagnoses. As observed by Kennedy et al. [22], with the lack of current literature regarding the effect of comorbid mTBI and PTSD, we can only speculate about how these two illnesses interact to affect attentional function. Recent studies have shown that the presence of PTSD appears to hinder the recovery from TBI [23]. Also, the areas of the brain that appear to be most vulnerable to TBI are also the same areas that are related to dysfunction in PTSD patients [22]. However, demand is increasing for research that investigates the clinical and therapeutic implications of a shared diagnosis and how to best treat troops returning from their service. To our knowledge, no study has been conducted that investigates attentional performance in combat veterans with comorbid mTBI and PTSD. In addition, while many studies have shown attentional deficits following TBI, most have studied severe TBI patients or mTBI patients with relatively recent injuries; only one study we are aware of [9] utilized a method enabling the discovery of hemispheric asymmetries in attentional performance. Furthermore, cognitive symptom resolution could also present an issue, considering that symptoms may have resolved in some mTBI cases but remain in others. The present study examines the extent to which attentional performance deficits exist in returning veterans from the Iraq and Afghanistan wars with relatively older mTBI injuries (2-6 yr) and PTSD comorbidity. Since the comorbidity of mTBI and PTSD is common in Department of Veterans Affairs (VA) medical centers (VAMCs), we decided to compare the attentional performance of this mTBI+PTSD patient group with a group of returning veterans with PTSD only and a control group of nondisabled veterans. Ideally, a cohort of patients with mTBI alone was desired for participation in this study; however, this was not possible because of the lack of veterans with a sole diagnosis of mTBI at the study site. In fact, of the 47 participants recruited for this study, not 1 had a sole diagnosis of mTBI. Therefore, comparing the performance of the mTBI+PTSD group and the PTSD group will provide us with the opportunity to distinguish between the attentional deficits caused by PTSD versus those caused by a dual diagnosis of mTBI+PTSD. We will use a lateralized version of the ANT (LANT) as a behavioral measure of attentional function. This computerized visual attention task measures the speed and accuracy with which subjects can shift attention to locations in the visual field (VF) and selectively focus attention on target stimuli. The LANT was designed to measure the efficiency of the alerting, orienting, and executive attentional networks. Since our method selectively flashes stimuli to the RVF and LVF on a given trial, hemispheric asymmetries in attentional performance can also be measured. The results of this study will help to establish quantitative profiles of attentional performance in those with an isolated PTSD diagnosis versus those with comorbidity of PTSD and mTBI. 


\section{METHODS}

\section{Participants}

A total of 47 combat veterans between the ages of 19 and 45 who served in OIF and/or OEF were recruited from the Charles George VAMC in Asheville, North Carolina, to participate in this study. The participants were grouped according to diagnosis: (1) 17 were diagnosed with PTSD without mTBI, (2) 15 with both mTBI and PSTD, and (3) and 15 with neither diagnosis. The participants had no history of a previous ocular disease or ADHD (before military deployment) or active drug/alcohol abuse or dependencies. The participants were also capable of giving informed consent as determined by the investigator's clinical judgment. Participants were screened by examination of their medical record in the Computerized Patient Record System (CPRS) to determine which best met inclusion criteria. Eligibility determination for using CPRS was approved by the institutional review board (IRB). A Waiver of Informed Consent for screening through CPRS met approval criteria for 45 CFR 46.116 (d) (1) no more than minimal risk; (2) will not adversely affect the rights and welfare of the subjects; (3) could not practicably be carried out without the waiver; and (4) whenever appropriate, the subjects will be provided with additional pertinent information. A HIPAA (Health Insurance Portability and Accountability Act of 1996) Waiver of Authorization to conduct the study met approval criteria 45 CFR 164.512 (i)(2)(ii) through the IRB and the Office of Research and Development.

Historical diagnoses from the participant's medical record were used to identify an mTBI and/or PTSD diagnosis. The key factors in diagnosing a case of mTBI include an injury event, such as a blow to the head, which causes an alteration of consciousness. The Defense and Veterans Brain Injury Center (DVBIC) has devised a 3question DVBIC TBI screening tool to help identify veterans who may be experiencing a TBI. A patient screens positive if he or she endorses an injury (Question 1), as well as an alteration of consciousness (Question 2 A-E). The mTBI screen alone does not provide a diagnosis of mTBI. If after a clinical interview the veteran screens positive, he or she is offered a comprehensive evaluation by a Component II or a Component III polytrauma team. These teams conduct a more thorough neuropsychological evaluation to determine whether the veteran has mTBI. The diagnosis of mTBI in our subjects was based on second-level evaluations from a Component II or III polytrauma team. A diagnosis of PTSD at a VAMC is similar to the TBI screen. A veteran is asked a series of questions at his or her initial appointment. The following healthcare providers can perform initial examinations for PTSD: board-certified psychiatrist, licensed doctoratelevel psychologist, doctorate-level mental health provider, psychiatry resident, or clinical psychologist completing a 1-year internship or residency. If the veteran screens positive, he or she is offered a clinical interview in which the mental health professional determines whether the veteran meets criteria that conform to the diagnostic criteria established in the Diagnostic and Statistical Manual of Mental Disorders, 4th edition, Text Revision [24]. The diagnosis of PTSD in our participants was based on a clinical evaluation by a mental health professional. The participant's most recent depression screen, the Patient Health Questionnaire-2 (PHQ-2), was also reviewed. The PHQ-2 asks two questions: (1) whether the patient has had little interest or pleasure in doing things and (2) whether the patient has been feeling down, depressed, or hopeless over the past 2 wk. The PHQ-2 is used not to diagnose depression but rather to screen for depression and determine the frequency of recent depressed mood. The scores range from 0 to 6 ; if the score is 3 or higher, it indicates a positive screen and suggests the need for further evaluation. Because the PHQ-2 is a depression screen and not a depression measure, the participant groups are not comparable in depression severity.

Participant inclusion criteria included combat veterans who had served in OIF and/or OEF; were between the ages of 19 and 45; and had a diagnosis of mTBI and PTSD, PTSD only, or neither. Exclusion criteria were active drug/alcohol abuse or dependence, prior diagnosis of an ocular disease, or prior diagnosis of an attention disorder. All the participants in the mTBI+PTSD and PTSD groups were actively engaged in rehabilitation and treatment at the time of this study. The participant's age, sex, most recent depression screen, duration of diagnosis, and type of blast exposure were also examined to determine whether the groups were comparable with respect to these variables. Table 1 summarizes the characteristics of participants in this study. All three groups were statistically similar in regards to participant characteristics.

\section{Instruments}

The LANT is a modified version of the original ANT developed by Fan et al. [12]. This task has been proven to be a useful tool in attention research because of its simplicity and reliability [12]. The LANT presents stimuli selectively to either the LVF (RH) or RVF (left 
Table 1.

Characteristics of participants in study $(n=47)$ with both mTBI and PTSD (mTBI+PTSD), PTSD only, or neither (control). Data shown as mean \pm standard deviation or $n(\%)$.

\begin{tabular}{lccc}
\hline \multicolumn{1}{c}{ Characteristic } & \multicolumn{3}{c}{ Group } \\
\cline { 2 - 4 } & mTBI+PTSD & PTSD only & Control \\
\hline Age (yr) & $30.41 \pm 8.60$ & $28.33 \pm 4.67$ & $29.00 \pm 4.29$ \\
PHQ-2 Depression Negative Score & $12(71)$ & $11(73)$ & $15(100)$ \\
Time Since PTSD Diagnosis (mo) & $18.35 \pm 15.75$ & $12.07 \pm 8.39$ & - \\
Time Since mTBI (mo) & $48.65 \pm 15.74$ & - & - \\
No. of Blasts & $1.65 \pm 0.79$ & - & - \\
Type of Blast Event & & & - \\
Primary & $23(82)$ & - & - \\
Secondary & $3(11)$ & - & - \\
Tertiary & $2(7)$ & - & - \\
\hline
\end{tabular}

*Primary = injury caused by blast overpressure, secondary = injury caused by bomb fragment, tertiary = injury caused by displacement of air from explosion. mTBI = mild traumatic brain injury, PHQ-2 = Patient Health Questionnaire-2, PTSD = posttraumatic stress disorder.

hemisphere [LH]). The targets are preceded by one of four cue types: no cue, valid spatial cue (presented at the location of the upcoming target), central cue, and double cue (presented at the two possible locations of the target). Subjects are instructed to fixate on a centrally located crosshair in the middle of the display screen and remain alert to the presentation of the spatial cues and target. Upon presentation of the target, the subject's task is to respond as rapidly as possible as to the orientation of the target (up or down). Each target is accompanied by flanker arrows that point either in the same direction as the target (congruent) or in the opposite direction (incongruent).

Following the standard approach [12], three metrics were calculated to measure the efficiency of the alerting, executive, and orienting networks. The efficiency metric for the alerting network was calculated by subtraction of the mean response time (RT) for the double cue condition from the no cue condition. The orienting efficiency metric was determined by subtraction of the mean RT for the spatial cue condition from the mean RT for the center cue condition. And the executive metric was calculated by subtraction of the mean RT for congruent flanker trials from the mean RT of incongruent flanker trials. In summary, the following formulas were used to calculate efficiency metrics for the three attention networks:

$$
\begin{gathered}
\text { Alerting Metric }=R T_{\text {no cue }}-R T_{\text {double cue }} \\
\text { Orienting Metric }=R T_{\text {center cue }}-R T_{\text {spatial cue }}
\end{gathered}
$$

Executive (Conflict) Metric $=R T_{\text {incongruent flanker }}-R T_{\text {congruent flanker }}$

\section{Apparatus and Stimuli}

The experiment was conducted in an examination room at a military medical center. The stimuli were presented on a Dell (Round Rock, Texas) Inspiron 1525 computer attached to a 19 in. 1703FPs Dell monitor with a refresh rate of $60 \mathrm{~Hz}$ and a resolution of 1,280 $\times$ 1,024 pixels. Participants viewed the screen from a distance of $65 \mathrm{~cm}$ and responses were collected from a Dell keyboard placed in front of the participants at midline. Participants were instructed to focus on the crosshair symbol that appeared in the center of the screen at the beginning of each trial, maintain fixation there throughout the trial, and to respond as quickly as possible while being reasonably sure that the response was accurate.

Target and associated flanker arrows pointed up or down and were presented to the right or left of the participant's fixation (Figure 1). The stimulus array (i.e., target and flanker arrows) spanned $2.9^{\circ}$ of visual angle and each arrow was $0.5^{\circ}$ long and $0.07^{\circ}$ wide. The head of the arrow was $0.15^{\circ}$ long and wide. There was a $2.2^{\circ}$ distance between the target and the fixation crosshair. Targets were preceded by one of four cue conditions: no cue, spatial cue (presented at the location of the target), central cue (presented at the fixation point), and double cue (presented at the two possible locations of the target). Stimuli consisted of a row of five vertical black lines with arrowheads pointing upward or downward against a white background. The central target arrow was flanked above and below by two arrows in the same direction (congruent flankers), two arrows in the opposite direction (incongruent flankers), or line segments with no arrow head (neutral flankers). The sequence of events for each 


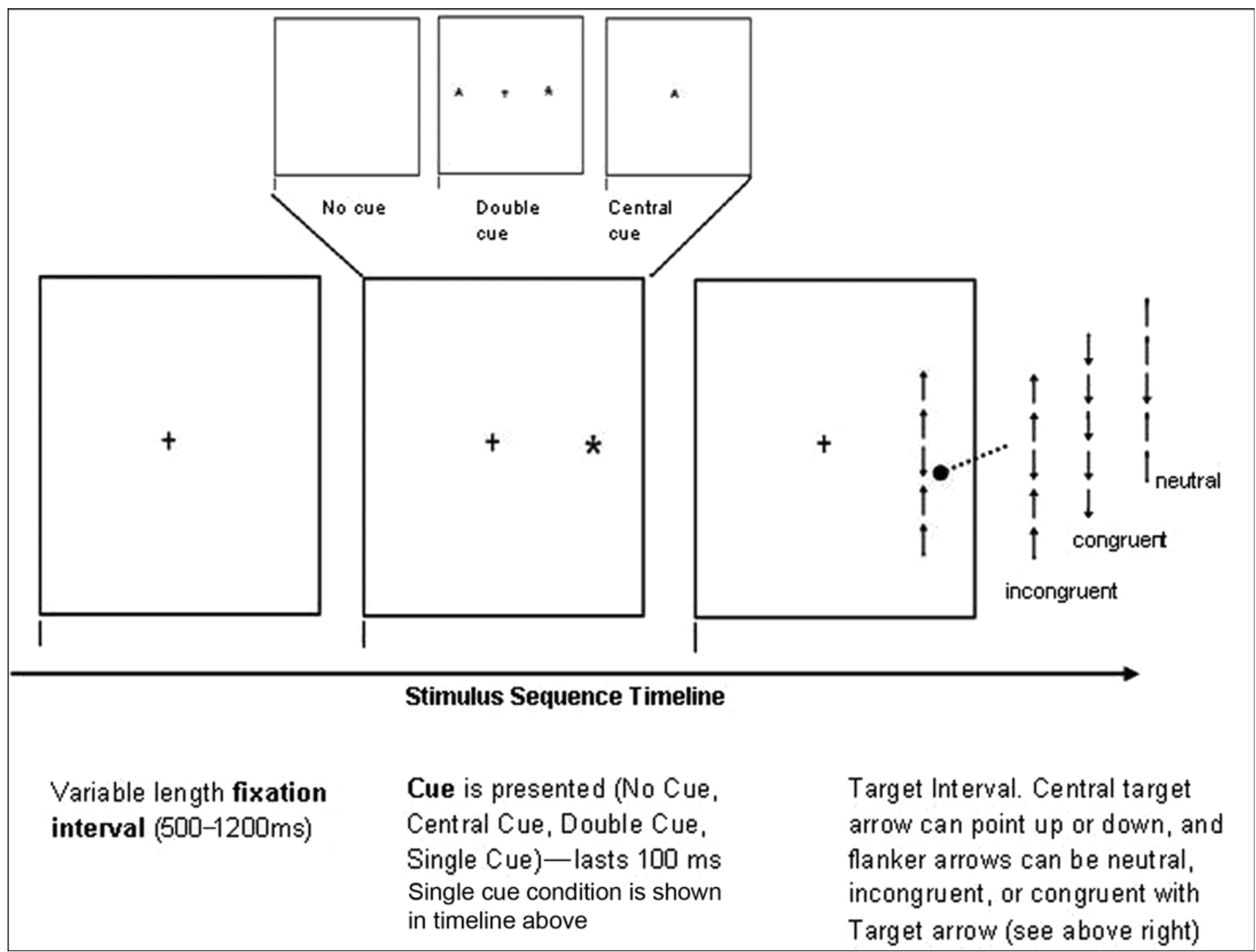

Figure 1.

Visual stimuli and timeline of attention network task stimulus sequence.

trial began with the fixation point (ranged from 500 to $1,200 \mathrm{~ms}$ ), followed by one of the cue types (presented as a red asterisk symbol for $100 \mathrm{~ms}$ ), a $150 \mathrm{~ms}$ delay, and then the target and flanker stimuli (300 ms). The target array was presented in the LVF and RVF on 1/2 the trials. Likewise, the orientation of the target arrow was equally divided between up and down across trials, $1 / 4$ of the trials presented each of the four cue types (no cue, central cue, double cue, and spatial cue), and 1/3 of the trials presented each of the flanker types (congruent, incongruent, neutral). The order of cue type $\times \operatorname{VF} \times$ target orientation $\times$ flanker type combinations was uniquely randomized for each participant, yielding a total of 96 trials per participant.

\section{Procedure}

Participants completed two screens measuring vision and attention. Additionally, they were asked whether they were experiencing any problems with vision or attention after returning from deployment. A thorough examination of the participants' visual functioning was measured to determine whether they had any ophthalmic impairment, i.e., diplopia or low visual acuity, that would interfere with the attention screen. During the visual screening process, the participants' visual acuity was measured using Snellen notation and intraocular pressure was obtained by air tonometry. The participants' current prescription was assessed, and they were refracted to their 
best visual acuity. All participants who participated in the study had a best corrected visual acuity of 20/30 or better with both eyes at distance and near. The vision screen was followed by the LANT. Before the task, the directions were explained to the participants, informing them to maintain fixation on the centrally located fixation cross and to try and remain alert throughout the trials. They were informed that the goal was to determine the orientation of the central target arrow (up or down) and press the corresponding arrow on the keyboard as quickly as possible. Participants were instructed to place their left index finger on the "up" arrow key and their right index finger on the "down" arrow key. Participants were initially administered a 12-trial practice block in which they received immediate feedback for their accuracy. Following the practice block, the participants were administered the experimental block of the LANT (96 trials). The 96 trials were uniquely randomized for each participant and did not give any visual feedback. To address possible problems with fatigue, participants were advised that they could take a break during the trials of the LANT and should tell the study coordinator if they needed one. They were also given a break after the trial block was administered. RT and response accuracy were the dependent variables. We also calculated and analyzed the three metrics of attentional network efficiency following Fan et al.'s [12] approach.

\section{RESULTS}

Mean RT, response accuracy, and the RT standard deviation (SD) values are presented in Table 2 and Figure 2. RT SD measures the intrasubject variability of RT across the trials of the LANT. We performed a mixed two-way analysis of variance (ANOVA) on these three measures, with patient group (mTBI+PTSD, PTSD, control) and VF (LVF, RVF) being the independent variables. Patient group had main effects on RT $(F(2,44)=6.9, p=$ $\left.0.002 ; \eta^{2}=0.25\right)$ and intrasubject variability of RT $(F(2$, $44)=3.8, p=0.03 ; \eta^{2}=0.17$ ). Post hoc analyses revealed that the mTBI+PTSD group's performance for each of these metrics was significantly worse than both the PTSD and control groups' performances (all type I probabilities for these analyses were $<0.05$ ), but no differences were found between the PTSD and control groups. The patient group factor did not have a significant effect on performance accuracy $(F(2,44)=2.2, p=$ 0.13 ), although the pattern of accuracy score was consistent with the other measures, showing poorer performance for the mTBI+PTSD group (Figure 2). VF did not have a significant effect on any of the metrics, and no interaction was found between VF and patient group.

In order to determine the effects of VF and patient group factors on the three attentional functions measured by the LANT, we performed three separate ANOVAs on the alerting, orienting, and executive metrics of attention using VF and patient group as the independent variables. A significant $\mathrm{VF} \times$ patient group interaction was found

Table 2.

Average response times, accuracy scores, and response time SD for participants with both mTBI and PTSD (mTBI+PTSD), PTSD only, or neither (control).

\begin{tabular}{|c|c|c|c|}
\hline \multirow{2}{*}{ Variable } & \multicolumn{3}{|c|}{ Mean } \\
\hline & LVF & RVF & Combined \\
\hline \multicolumn{4}{|l|}{ Response Time (ms) } \\
\hline mTBI+PTSD & 989 & 982 & 1,971 \\
\hline PTSD & 778 & 755 & 1,533 \\
\hline Control & 668 & 660 & 1,328 \\
\hline \multicolumn{4}{|l|}{ Accuracy (\% Correct) } \\
\hline mTBI+PTSD & 80.1 & 80.4 & 80.3 \\
\hline PTSD & 85.6 & 84.8 & 85.2 \\
\hline Control & 88.1 & 88.9 & 88.5 \\
\hline \multicolumn{4}{|c|}{ Intrasubject Response Time SD (ms) } \\
\hline mTBI+PTSD & 476 & 530 & 1,006 \\
\hline PTSD & 379 & 288 & 667 \\
\hline Control & 221 & 221 & 442 \\
\hline
\end{tabular}




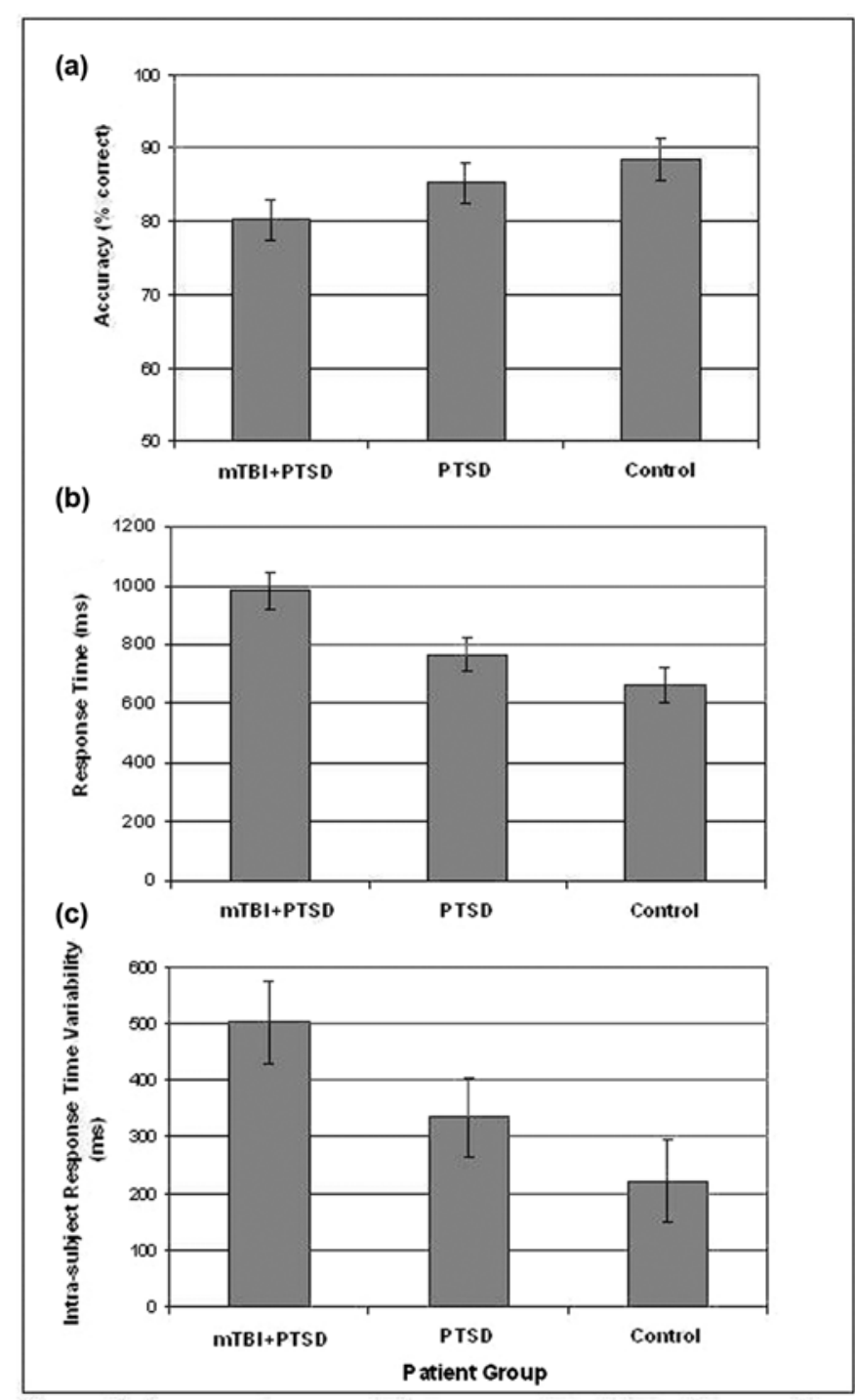

Figure 2.

(a) Average response accuracy, (b) response time, and (c) intrasubject variability in response time for mild traumatic brain injury $(\mathrm{mTBI})+$ posttraumatic stress disorder (PTSD), PTSD only, and control groups.

for the orienting metric $\left(F(1,44)=3.14, p=0.05 ; \eta^{2}=\right.$ 0.14). mTBI+PTSD participants exhibited no RT benefit of spatial cues presented in the LVF but a substantial benefit in the RVF (Figure 3); the PTSD group exhibited the opposite asymmetry, showing more benefit in the LVF than $\operatorname{RVF}$ ( $p \leq 0.05$ for pairwise comparisons). This finding suggests that mTBI+PTSD participants had more difficulty covertly shifting attention to the LVF using RH mechanisms, whereas the PTSD participants had more difficulty shifting attention to the RVF using LH mechanisms. We found no main or interaction effects for the alerting and executive metrics of attention.

Before the study onset, we asked the participants whether they had noticed any problems with attention or concentration following the traumatic event. Based on their answers, we grouped the participants into two categories: no complaint and complaint. Similar to the Lew et al. study [5], 47 percent of the veterans self-reported attentional problems in everyday activities. We found a significant $\mathrm{VF} \times$ patient group interaction effect on accuracy scores $\left(F(1,14)=11.0, p=0.005 ; \eta^{2}=0.44\right)$, indicating that that participants who complained of attention problems performed significantly worse than the no-complaint participants when stimuli were projected to the LVF, suggesting an RH performance deficit (Table 3, Figure 4). The RT measure showed the same pattern of performance $\left(F(1,14)=4.4, p=0.056 ; \eta^{2}=0.24\right)$ : the complaint participants were slower to respond than the no-complaint participants, but only when stimuli were presented in the LVF.

\section{DISCUSSION}

While our study did not reveal any significant differences in attentional performance between PSTD participants

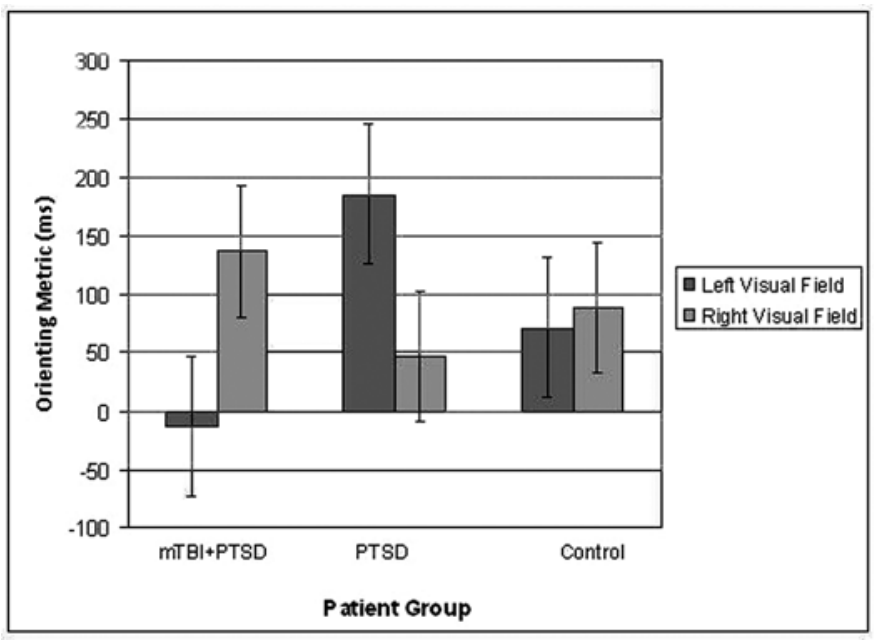

\section{Figure 3.}

Orienting efficiency. Response time benefit of preceding target with spatial cue for mild traumatic brain injury (mTBI) + posttraumatic stress disorder (PTSD), PTSD only, and control groups. 
and control subjects, clear trends in the data showed that PTSD participants were somewhat slower, less accurate, and more variable in RT from one trial to the next than controls (Figure 2). These trends are consistent with other studies showing attentional deficits with PTSD [20-21,25-26]. Consistent with prior studies [6,9-10], attentional performance was substantially slower and less accurate for mTBI+PTSD participants than for the other participant groups (Figure 2). We also observed substantial individual differences in performance across participants in the mTBI+PTSD group. Some mTBI+PTSD participants performed equal to or better than the control group as a whole, while others performed substantially worse. We speculate that this high variability might be related to large individual differences associated with mTBI. These differences may include size of blast, proximity of blast, type of blast, and length of time since injury. The intrasubject variability of mTBI+PTSD participant responses was also substantially higher than for the PTSD and control groups (Table 2, Figure 2). This could indicate that the mTBI+PTSD group had trouble maintaining attentional focus and, as a result, manifested substantial lapses of attention across trials of the LANT, which caused a large variability in RTs. The significantly slower attentional performance of mTBI+PTSD participants and their more variable RTs might be related to reduced information-processing speed, thought to be caused by diffuse axonal injury [27]. Attentional dysregulation may also be due to the synergistic effects of both mTBI and PTSD. Additionally, we did not measure PTSD symptom severity at the time of testing, which could indicate that the variability in the mTBI+PTSD group could be due to group differences in PTSD severity and not mTBI.

\section{Table 3.}

Lateralized attention network task accuracy scores (\% correct) for participants with both mTBI and PTSD diagnoses.

\begin{tabular}{|c|c|c|}
\hline \multirow[t]{2}{*}{ Group } & \multicolumn{2}{|c|}{$\begin{array}{c}\text { Mean } \pm \text { Standard Deviation } \\
\% \text { Correct }\end{array}$} \\
\hline & LVF & RVF \\
\hline $\begin{array}{l}\text { Did Not Report Attention } \\
\text { Problems }\end{array}$ & $83.3 \pm 12.0$ & $79.1 \pm 12.0$ \\
\hline Reported Attention Problems & $69.1 \pm 19.0$ & $75.0 \pm 23.0$ \\
\hline
\end{tabular}

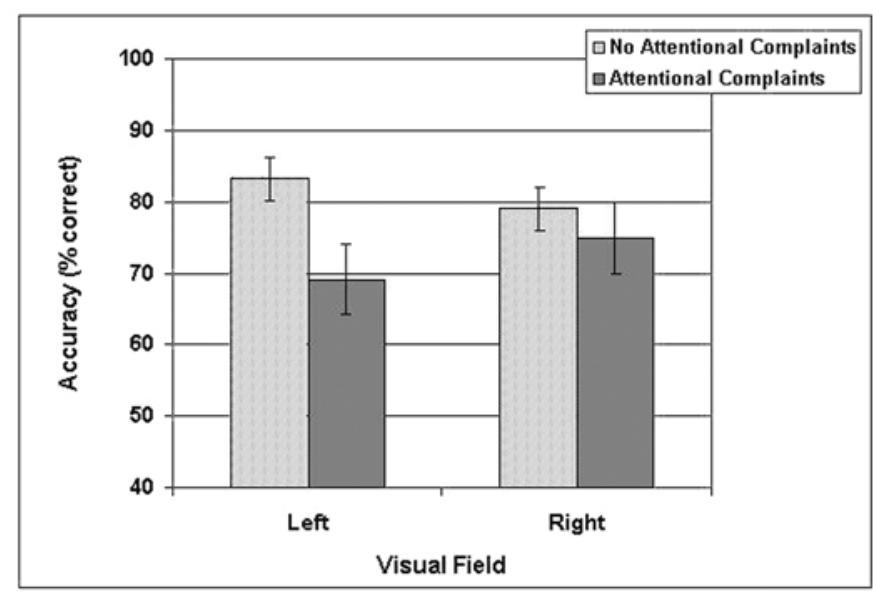

Figure 4.

Response accuracy for participants with mild traumatic brain injury who self-reported attentional complaints versus those who did not report such complaints.

The orienting component of attention involves the ability to spatially disengage the focus of attention from one spatial location and shift to another location. Numerous studies have found that when a spatial cue to target location is presented just before the onset of the target stimuli, RTs are faster than without a spatial cue, presumably because the respondent is able to covertly disengage and shift attention to the target location before its appearance, thus speeding the analysis of target information. In our study, we found that the mTBI+PTSD group took significantly longer and was less accurate than the PTSD and control groups when a spatial cue and target were presented to the LVF, presumably indicating they were less efficient in disengaging and shifting spatial attention to the LVF (thus indicating an $\mathrm{RH}$ deficit in orienting attention). Our finding that mTBI affects the orienting function of attention is consistent with several other studies. In a study involving civilian patients who had experienced a concussion, Van Donkelaar et al. measured attentional deficits with the ANT [10]. Their results suggested that the orienting and executive components of attention were the most susceptible to the effects of concussion, while the alerting component remained intact. They also found overall slower RTs and poorer accuracy for TBI patients, similar to the results of our study. While our results were not significant, the pattern of data we observed for this metric was consistent with Van Donkelaar et al.'s [10] finding (RT cost of incongruent flankers 
in our study was $145 \mathrm{~ms}$ for mTBI+PTSD group and $73 \mathrm{~ms}$ for controls). In another study, Pavolvskaya et al. also found that TBI patients exhibited a significant asymmetry in orienting attention [9]. Specifically, they were less efficient at shifting their visual attention to the LVF. We observed the same pattern in our study, in so far as the metric measuring orienting efficiency was substantially smaller in the LVF than the RVF for mTBI+PTSD participants. The PTSD group in our study exhibited the opposite asymmetry in orienting attention, showing substantially better performance in the LVF than RVF. This finding suggests that while mTBI+PTSD participants had more difficulty shifting attention to the LVF using RH mechanisms, the PTSD participants had more difficulty shifting attention to the RVF using LH mechanisms. The latter asymmetry observed with the PTSD participants is consistent with the results of another study of Vietnam veterans diagnosed with PTSD [17]. In that study, subjects were required to attend to global versus local aspects of stimulus patterns; the PTSD subjects were found to be significantly slower in identifying local versus global targets, whereas the control subjects were faster in identifying local targets. Given that the RH tends to be specialized for global perception and the $\mathrm{LH}$ for local perception, the results provide at least partial support for the hypothesis of an LH deficit in PTSD. This hypothesis is also supported by other behavioral studies and brain imaging studies indicating hypoactivation of the LH in PTSD [28-29]. The observation that the mTBI+PTSD participants and the PTSD participants exhibited different hemispheric asymmetries in orienting attention suggests that mTBI was the dominating morbidity driving the $\mathrm{RH}$ attentional deficit in the $\mathrm{mTBI}+\mathrm{PTSD}$ group. However, we can only speculate that the mTBI caused the hemispheric asymmetry, given the lack of an isolated mTBI group.

While other studies involving PTSD subjects have shown attentional deficits [20-21,26], we found no major differences between military PTSD subjects and controls in our study on behavioral measures of attention. We were unable to replicate the findings of Leskin and White's study that suggested that PTSD participants were slower in responding to cues presenting conflicting information, the executive network, when compared with nonPTSD participants [20]. One of the reasons for this difference may have been that we did not measure current PTSD symptom severity in our study. Leskin and White noted that elevated PTSD symptoms produced an effect on ANT measures of executive functioning [20]. PTSD participants were slower at responding to cues that presented conflicting stimuli. We may have been unable to replicate Leskin and White's [20] findings that higher PTSD symptom severity may be associated with executive function deficits because our participants were actively engaged in treatment and rehabilitation and, thus, not experiencing elevated PTSD symptoms. However, we can only speculate this was the cause because we did not measure current PTSD symptom severity.

Nearly half our mTBI+PTSD cohort self-reported attentional problems in everyday activities in their preexperiment interview. When we compared performance on our behavioral measures of attention between those participants reporting attentional problems versus those not reporting such problems, we found an interesting hemispheric asymmetry. Participants reporting attention problems performed significantly worse in the LVF than participants not reporting attention problems, suggesting an RH attention deficit (Figure 4). These results are consistent with Poynter et al.'s study [14], which showed that nondisabled subjects who self-reported relatively high levels of attention problems on the Conner's ADHD scale performed significantly worse on two behavioral measures of attention when stimuli were presented in the LVF versus RVF. This result was most prominent in the condition with spatial cues, indicating that deficits in orienting attention to the LVF (using RH mechanisms) tend to correlate with self-rated attention problems. This $\mathrm{RH}$ deficit has been documented in various studies of ADHD, and right parietal lobe damage is widely documented to produce more severe attentional neglect than left parietal lobe damage [30-32]. Our results and those of Pavlovskaya et al. [9] provide initial evidence that at least subgroups of mTBI+PTSD patients might have dysfunctions of the RH parietal lobe and prefrontal cortex, based on brain imaging studies of anatomical loci involved in the orienting of visual attention [33].

Properly diagnosing and determining the proper clinical care and treatment for our servicemembers who have experienced mTBI are challenging for several reasons. Initially, blast-related mTBI does not manifest observable neuroanatomical lesions because injury is diffuse and does not selectively injure a specific region of the brain. Also, most of the current literature pertaining to mTBIs is civilian related. Can you adequately compare the civilianversus combat-related mTBI literature? Combat-related mTBI might be considered different from sports-related 
or fall- and/or accident-related head injury because of the context of the injury (e.g., blast event vs car accident) and the damage of injury (e.g., diffuse vs localized). The extent of differences/similarities in cognitive dysfunction related to civilian- versus combat-related mTBI is not well understood; however, the results of our study suggest substantial similarities in attentional performance deficits. Overall, considering the high comorbidity of mood and anxiety disorders with mTBI and the lack of evidence suggesting the long-term cognitive deficits following mTBI, attributing any of the results of our study to an isolated diagnosis of mTBI is difficult; rather, our results contribute to the literature regarding the effect of comorbid diagnoses of mTBI and PTSD on behavioral measures of attention.

Regarding rehabilitation and clinical care of our veterans who have a diagnosis of mTBI+PTSD, some studies [34] have suggested the need for long-term intervention with patients after a TBI. Olver et al. examined long-term outcomes of individuals with a TBI following discharge from a rehabilitation program [34]. The results of their study indicate that 5 yr after completing the rehabilitation program, individuals with TBI were still experiencing neurological symptoms; problems with mobility, independence in activities of daily living productivity, and relationships; and cognitive, behavioral, and emotional changes. Olver et al.'s [34] results suggest the need for perhaps lifelong intervention following TBI. Consistent with the results from their study, we found that the attentional effects of mTBI are still present years after the event. The long-term functional outcomes associated with mTBI can be complex and challenging in regard to rehabilitation and clinical care. The results of our study, as well as Olver et al.'s [34], suggest that patients who have sustained a TBI may still experience symptoms years after the event. With respect to clinical care, these patients should be monitored over an extended period of time and clinicians should view the diagnosis as a potential long-term disability. However, more literature is needed to address the average recovery time of TBI compared with mTBI to determine whether the rehabilitation and clinical care needed significantly differ. In addition, the observations made in this study were seen only among veterans with mTBI and PTSD and it is unclear whether the subtle attentional impairments are due to the neurological or psychiatric issues. Overall, these results may help update rehabilitation approaches and development of interventions and guide future research related to individuals who have sustained an mTBI and PTSD.

\section{LIMITATIONS OF STUDY AND IMPLICATIONS FOR FUTURE RESEARCH}

The large variability in attentional performance within the mTBI+PTSD group might be explained by several factors. We had no measure of premorbid attentional function, so some of the mTBI+PTSD veterans may have exhibited slower and less accurate attentional performance before their blast event. In addition, the exact time line regarding the changes in neuropathology recovery after mTBI is unclear. Research suggests that the progression of recovery may range from days to months after exposure to a blast event [35]. However, some symptoms may remain years after the event. Within our mTBI+PTSD sample, exposure to the blast event occurred between 2 and $7 \mathrm{yr}$ before the testing. This wide range of recovery time after injury may help to explain the variability in attentional performance with the military TBI patient population. The severity of the blast event also needs to be considered when working within this population. The VA and Department of Defense define blast events as primary, secondary, tertiary, and quaternary. Blast events can occur from the overpressure postexplosion or from direct impact to the head. The etiology of the injury also ranges from improvised explosive devices to mortar, shrapnel, grenade, and vehicular. Further investigation that distinguishes attentional performance between these types of head injuries is needed. Furthermore, the non-mTBI groups may have experienced a predeployment TBI. The DVBIC TBI screening tool asks questions that specifically pertain to injuries that occurred during deployment. Because nonmilitary TBIs were not assessed, this could be another potential limitation of the study. Patients with mTBI are also often prescribed certain medications that help alleviate the side effects common to injury. For example, Topamax is often given for relief of migraines. The degree to which these medications might affect attentional function is unclear. The individuals with coexisting diagnoses of PTSD and mTBI may also exhibit greater severity of PTSD than those with PTSD only. PTSD symptoms were not measured at the time of testing in our study, so we are unable to determine the effect of mood and symptom severity on attention. Thus, there may have been potential group 
differences in attentional performance related to symptom severity. Future studies in which a group of subjects with a sole diagnosis of mTBI is used and in which symptom severity of PTSD is measured at testing would be beneficial to attentional research. Considering the high intrasubject variability found in the mTBI+PTSD group, these differences may be expected based on the rehabilitation status of each participant. Although all the participants in the PTSD and mTBI+PTSD groups were actively receiving treatment at the time of the study, some patients may not adhere to rehabilitation regimens and recommendations. In effect, the results may have been influenced by the difference in rehabilitation status within our patient population. Another potential limitation is that the groups may have varied in level of depression. The PTSD and mTBI+PTSD groups were the only participants that endorsed depressive symptoms as measured by the PHQ-2. However, depression level was not measured, which could have also influenced our findings.

Although a diagnosis of ADHD was an exclusion criterion for our study, 3 of the $17 \mathrm{mTBI}+\mathrm{PTSD}$ patients were recently diagnosed with an attentional impairment. These patients observed no attentional problems before their blast event. Clinical implications need to be considered when diagnosing these individuals with ADHD. Future research is needed to investigate the attentional complaints that differentiate mTBI from ADHD for optimal rehabilitation and medical care. The large intrasubject variability in attentional performance among our mTBI+PTSD group perhaps indicates that these participants experienced lapses of attention or were more easily distracted than the PTSD or control participants. Consistent with this idea, Segalowitz et al. concluded that variability in attention task RT is the most valid measure of TBI patients' ability to sustain attention and that TBI patients exhibit an inability to maintain focus throughout the duration of attention tasks [36]. Future studies using larger mTBI sample sizes and more sensitive attentional measures of the alerting, orienting, and executive networks of attention may contribute to a better understanding of the effects of mTBI on attentional function, hemispheric asymmetries in these effects, and the time course of recovery from mTBI injuries.

\section{CONCLUSIONS}

This study examined the attentional effects of mTBI and PTSD in a U.S. military veteran cohort to help guide clinical care and rehabilitation practices. As for attentional abilities, we conclude that a diagnosis of mTBI+PTSD does cause impairment in attentional function, in some cases years after the date of the injury. In regard to clinical care, our results indicate that rehabilitation practices should consider a diagnosis of mTBI+PTSD as a possible long-term injury. In addition, it remains unclear whether our findings were due to the neurological or psychiatric impairments within this population. Consistent with a number of previous studies, we found that our group with a diagnosis of mTBI+PTSD was substantially slower and more variable in its attentional responses. Additionally, we found partial evidence for hemispheric asymmetry in attentional function following combat-related TBI and PTSD, with mTBI+PTSD participants showing greater deficits in RH function and PTSD only participants showing greater deficits in LH function.

\section{ACKNOWLEDGMENTS}

\section{Author Contributions:}

Study concept and design: K. Barlow-Ogden, W. Poynter.

Acquisition of data: K. Barlow-Ogden.

Analysis and interpretation of data: K. Barlow-Ogden, W. Poynter.

Drafting of manuscript: K. Barlow-Ogden, W. Poynter.

Critical revision of manuscript for important intellectual content:

K. Barlow-Ogden, W. Poynter.

Statistical analysis: K. Barlow-Ogden, W. Poynter.

Administrative, technical, or material support: K. Barlow-Ogden, W. Poynter.

Study supervision: K. Barlow-Ogden, W. Poynter.

Financial Disclosures: The authors have declared that no competing interests exist.

Funding/Support: This material is based on work supported by the VA, Veterans Health Administration, Office of Research and Development. This material is also the result of work supported with resources and the use of facilities at the Charles George VAMC in Asheville, North Carolina.

Additional Contributions: We would like to thank Lisa Bishop, OD, for her assistance in data collection and Art Horn, $\mathrm{PhD}$, for his assistance and support throughout this study. Ms. Barlow-Ogden is now also affiliated with the Department of Psychology, Fielding Graduate University, Santa Barbara, California.

Institutional Review: Eligibility determination for using CPRS was approved by the IRB. A Waiver of Informed Consent for screening through CPRS met approval criteria for 45 CFR 46.116 (d) and a HIPAA Waiver of Authorization to conduct the study met approval 
criteria 45 CFR 164.512 (i)(2)(ii) through the IRB and the Office of Research and Development.

Participant Follow-Up: The authors do not plan to inform participants of the publication of this study.

\section{REFERENCES}

1. Van Boven RW, Harrington GS, Hackney DB, Ebel A, Gauger G, Bremner JD, D’Esposito M, Detre JA, Haacke EM, Jack CR Jr, Jagust WJ, Le Bihan D, Mathis CA, Mueller S, Mukherjee P, Schuff N, Chen A, Weiner MW. Advances in neuroimaging of traumatic brain injury and posttraumatic stress disorder. J Rehabil Res Dev. 2009; 46(6):717-57. [PMID:20104401] http://dx.doi.org/10.1682/JRRD.2008.12.0161

2. Munsey C. A long road back. Mon Psychol. 2007;28(6):34.

3. Carson study: 1 in 6 show TBI symptoms. Associated Press, 2007 Apr 11.

4. Hoge CW, McGurk D, Thomas JL, Cox AL, Engel CC, Castro CA. Mild traumatic brain injury in U.S. soldiers returning from Iraq. N Engl J Med. 2008;358(5):453-63. [PMID:18234750] http://dx.doi.org/10.1056/NEJMoa072972

5. Lew HL, Poole JH, Guillory SB, Salerno RM, Leskin G, Sigford B. Persistent problems after traumatic brain injury: the need for long-term follow-up and coordinated care. J Rehabil Res Dev. 2006;43(2):vii-x. [PMID:16847779] http://dx.doi.org/10.1682/JRRD.2006.05.0054

6. Bate AJ, Mathias JL, Crawford JR. The covert orienting of visual attention following severe traumatic brain injury. J Clin Exp Neuropsychol. 2001;23(3):386-98.

[PMID:11404815] http://dx.doi.org/10.1076/jcen.23.3.386.1190

7. Cremona-Meteyard SL, Clark CR, Wright MJ, Geffen GM. Covert orientation of visual attention after closed head injury. Neuropsychologia. 1992;30(2):123-32. [PMID:1560891] http://dx.doi.org/10.1016/0028-3932(92)90022-E

8. Loken W, Thornton A, Otto R, Long C. Sustained attention after closed head-injury. Neuropsychology. 1995;9(4):592-98. http://dx.doi.org/10.1037/0894-4105.9.4.592

9. Pavlovskaya M, Groswasser Z, Keren O, Mordvinov E, Hochstein S. Hemispheric visual atentional imbalance in patients with traumatic brain injury. Brain Cogn. 2007; 64(1):21-29. [PMID:17182160]

http://dx.doi.org/10.1016/j.bandc.2006.10.003

10. Van Donkelaar P, Langan J, Rodriguez E, Drew A, Halterman C, Osternig LR, Chou LS. Attentional deficits in concussion. Brain Inj. 2005;19(12):1031-39. [PMID:16263646] http://dx.doi.org/10.1080/02699050500110363
11. Whyte J, Grieb-Neff P, Gantz C, Polansky M. Measuring sustained attention after traumatic brain injury: differences in key findings from the sustained attention to response task (SART). Neuropsychologia. 2006;44(10):2007-14. [PMID:16682059] http://dx.doi.org/10.1016/j.neuropsychologia.2006.02.012

12. Fan JI, McCandliss BD, Sommer T, Raz A, Posner MI. Testing the efficiency and independence of attentional networks. J Cogn Neurosci. 2002;14(3):340-47. [PMID:11970796] http://dx.doi.org/10.1162/089892902317361886

13. Halterman CI, Langan J, Drew A, Rodriguez E, Osternig LR, Chou LS, van Donkelaar P. Tracking the recovery of visuospatial attention deficits in mild traumatic brain injury. Brain. 2006;129(Pt 3):747-53. [PMID:16330498] http://dx.doi.org/10.1093/brain/awh705

14. Poynter W, Ingram P, Minor S. Visual field asymmetries in attention vary with self-reported attention deficits. Brain Cogn. 2010;72(3):355-61. [PMID:19931966]

http://dx.doi.org/10.1016/j.bandc.2009.10.014

15. Chokron S, Brickman AM, Wei T, Buchsbaum MS. Hemispheric asymmetry for selective attention. Brain Res Cogn Brain Res. 2000;9(1):85-90. [PMID:10666560] http://dx.doi.org/10.1016/S0006-8993(99)02169-1

16. Epstein JN, Conners CK, Erhardt D, March JS, Swanson JM. Asymmetrical hemispheric control of visual-spatial attention in adults with attention deficit hyperactivity disorder. Neuropsychology. 1997;11(4):467-73. [PMID:9345690] http://dx.doi.org/10.1037/0894-4105.11.4.467

17. Geeraerts S, Lafosse C, Vaes N, Vandenbussche E, Verfaillie K. Dysfunction of right-hemisphere attentional networks in attention deficit hyperactivity disorder. J Clin Exp Neuropsychol. 2008;30(1):42-52. [PMID:17852596] http://dx.doi.org/10.1080/13803390601186676

18. Catena RD, van Donkelaar P, Halterman CI, Chou LS. Spatial orientation of attention and obstacle avoidance following concussion. Exp Brain Res. 2009;194(1):67-77. [PMID:19082819] http://dx.doi.org/10.1007/s00221-008-1669-1

19. Godefroy O, Lhullier C, Rousseaux M. Non-spatial attention disorders in patients with frontal or posterior brain damage. Brain. 1996;119(Pt 1):191-202. [PMID:8624681] http://dx.doi.org/10.1093/brain/119.1.191

20. Leskin LP, White PM. Attentional networks reveal executive function deficits in posttraumatic stress disorder. Neuropsychology. 2007;21(3):275-84. [PMID:17484590] http://dx.doi.org/10.1037/0894-4105.21.3.275

21. Vasterling JJ, Duke LM, Tomlin H, Lowery N, Kaplan E. Global-local visual processing in posttraumatic stress disorder. J Int Neuropsychol Soc. 2004;10(5):709-18. [PMID:15327718] http://dx.doi.org/10.1017/S1355617704105031 
22. Kennedy JE, Jaffee MS, Leskin GA, Stokes JW, Leal FO, Fitzpatrick PJ. Posttraumatic stress disorder and posttraumatic stress disorder-like symptoms and mild traumatic brain injury. J Rehabil Res Dev. 2007;44(7):895-920. [PMID:18075948] http://dx.doi.org/10.1682/JRRD.2006.12.0166

23. Bryant RA, Marosszeky JE, Crooks J, Baguley IJ, Gurka JA. Posttraumatic stress disorder and psychosocial functioning after severe traumatic brain injury. J Nerv Ment Dis. 2001;189(2):109-13. [PMID:11225683] http://dx.doi.org/10.1097/00005053-200102000-00006

24. American Psychiatric Association. Diagnostic and statistical manual of mental disorders: DSM-IV. 4th ed. Washington (DC): American Psychiatric Association; 1994.

25. Jenkins MA, Langlais PJ, Delis DA, Cohen RA. Attentional dysfunction associated with posttraumatic stress disorder among rape survivors. Clin Neuropsychol. 2000; 14(1):7-12. [PMID:10855055]

http://dx.doi.org/10.1076/1385-4046(200002)14:1;1-8;FT007

26. Vasterling JJ, Duke LM, Brailey K, Constans JI, Allain AN Jr, Sutker PB. Attention, learning, and memory performances and intellectual resources in Vietnam veterans: PTSD and no disorder comparisons. Neuropsychology. 2002;16(1):5-14. [PMID:11853357]

http://dx.doi.org/10.1037/0894-4105.16.1.5

27. Madigan NK, DeLuca J, Diamond BJ, Tramontano G, Averill A. Speed of information processing in traumatic brain injury: modality-specific factors. J Head Trauma Rehabil. 2000;15(3):943-56. [PMID:10785624] http://dx.doi.org/10.1097/00001199-200006000-00007

28. Vasterling JJ, Rogers C, Kaplan E. Qualitative block design analysis in posttraumatic stress disorder. Assessment. 2000;7(3):217-26. [PMID:11037389] http://dx.doi.org/10.1177/107319110000700302

29. Shin LM, Kosslyn SM, McNally RJ, Alpert NM, Thompson WL, Rauch SL, Macklin ML, Pitman RK. Visual imagery and perception in posttraumatic stress disorder. A positron emission tomographic investigation. Arch Gen Psychiatry. 1997;54(3):233-41. [PMID:9075464]

http://dx.doi.org/10.1001/archpsyc.1997.01830150057010

30. Corbetta M, Patel G, Shulman GL. The reorienting system of the human brain: from environment to theory of mind.
Neuron. 2008;58(3):306-24. [PMID:18466742]

http://dx.doi.org/10.1016/j.neuron.2008.04.017

31. Heilman KM, Watson RT, Valenstein E. Neglect and related disorders. In: KM Heilman, E Valenstein, editors. Clinical neuropsychology. 3rd ed. New York (NY): Oxford University Press; 1993. p. 279-336.

32. Posner MI, Petersen SE. The attention system of the human brain. Annu Rev Neurosci. 1990;13:25-42. [PMID:2183676] http://dx.doi.org/10.1146/annurev.ne.13.030190.000325

33. Corbetta M, Shulman GL. Control of goal-directed and stimulus-driven attention in the brain. Nat Rev Neurosci. 2002;3(3):201-15. [PMID:11994752] http://dx.doi.org/10.1038/nrn755

34. Olver JH, Ponsford JL, Curran CA. Outcome following traumatic brain injury: a comparison between 2 and 5 years after injury. Brain Inj. 1996;10(11):841-48.

[PMID:8905161] http://dx.doi.org/10.1080/026990596123945

35. McCrea M, Guskiewicz KM, Marshall SW, Barr W, Randolph C, Cantu RC, Onate JA, Yang J, Kelly JP. Acute effects and recovery time following concussion in collegiate football players: the NCAA Concussion Study. JAMA. 2003;290(19):2556-63. [PMID:14625332] http://dx.doi.org/10.1001/jama.290.19.2556

36. Segalowitz SJ, Dywan J, Unsal A. Attentional factors in response time variability after traumatic brain injury: an ERP study. J Int Neuropsychol Soc. 1997;3(2):95-107. [PMID:9126851]

Submitted for publication September 22, 2010. Accepted in revised form February 6, 2012.

This article and any supplementary material should be cited as follows:

Barlow-Ogden K, Poynter W. Mild traumatic brain injury and posttraumatic stress disorder: Investigation of visual attention in Operation Iraqi Freedom/Operation Enduring Freedom veterans. J Rehabil Res Dev. 2012;49(7):1101-14. http://dx.doi.org/10.1682/JRRD.2010.09.0188

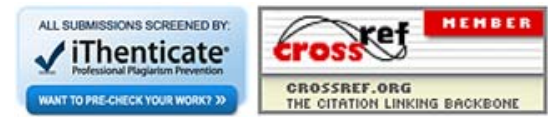

